\title{
The Relativistic Theory of Electromagnetic Interactions
}

\author{
A.V. Andreev ${ }^{a}$ \\ Physics Department, M.V. Lomonosov Moscow State University, Moscow 119992 Russia
}

Quantum - mechanical problem on electron motion in Coulomb field plays the fundamental role in the modern physics, because solution of this problem provides the most realistic model of atomic structure. In nowadays the model of meson atom is of the same importance. Indeed, the mesoatom is unstable, hence the study of interaction of mesons with atomic nuclei can provide the valuable information on the nature of hadronic interactions and structure of atomic nucleus.

The mesoatoms arise in the process of scattering of the negatively charged pions in matter. The pions captured by the target atoms populate initially the highly excited atomic orbits and then go down to $2 p$ and $1 s$ atomic states via cascade of radiative transitions. As far as the meson mass exceeds the electron mass for the two orders of magnitude then the mesonic atom orbit radius is much smaller than the radius of electron atom orbits. For example, the radius of the lowest mesonic orbits becomes comparable with the atomic nucleus size in the case of nucleus charge $Z \geq 12$ [1]. As a result, for $\pi^{-}$ meson in $2 p$ state the probability to annihilate on atomic nucleus is higher than the probability to decay radiatively in $2 p \rightarrow 1 s$ transition. In the atoms with the nucleus charge $Z \geq 40$ the radiative transitions disappear before the cascade reaches the $2 p$ state. In the case of atoms with more heavy nuclei even the $3 d$ states can not often be occupied. Thus the study of the cascade x-ray spectra of mesonic atoms provides the information on the efficiency of $\pi^{-}$meson interaction with the different nuclei.

The nonrelativistic Schredinger theory produces the atomic spectrum in zero-order approximation which does not include fine and hyperfine structure. The atomic spectrum calculated in the frames of the Dirac relativistic theory includes the fine structure. The hyperfine atomic structure can be calculated with the help of the invariant perturbation theory methods, which are based on the field quantization theory. The smallness parameter of invariant perturbation theory is the product of the hyperfine structure constant and nucleus charge, $Z \alpha$. Hence, the atoms with the nucleus charge of $Z \alpha \approx 1$ are out of the frames of the perturbation theory and therefore they are of special interest. This interest is due to a number of reasons:

Firstly, the precise mathematical calculations of hyperfine atomic structure can be based on the non-perturbative theory which is valid for arbitrary value of parameter $Z \alpha$.

Secondly, the Dirac theory predicts that at $Z \alpha \geq 1$ the energy eigenvalues become complex. It means that the corresponding atomic states are unstable, i.e. the lifetime of these states is finite [2-4].

\footnotetext{
${ }^{a}$ Corresponding author: av_andreev@phys.msu.ru
} 
Thus the question - what is the nature of the constraint $Z<Z_{0}=\alpha^{-1}$ ? - takes an extraordinary meaning. Evidently, that the answer this question can not be obtained in the frame of any perturbation theory.

Thirdly, to avoid the singularity of Coulomb potential at $r=0$ the cutoff Coulomb potential is used (see for example [5]). In this case the magnitude of parameter $Z \alpha$ for the stable atomic states can exceed the unity [5], therefore the concept of the critical nucleus charge is used to define the boundary of the stable atoms. The critical charge is defined as charge of nucleus at which the energy of $1 s$ atomic state becomes equal to $E_{1 s}=-m_{0} c^{2}$, i.e. when the energy of $1 s$ state reaches the "down" continuum. Notice, that in the frames of the cutted potentials the energy of atomic states falls usually with the nucleus charge [5]. However, this theory is inevitable based on the inclusion of new unknown parameter, which is the radius of cutting. The calculated spectra depend significantly on numerical value of this parameter.

It has been shown recently [6,7] that the quantum theory based on the wave equations including the second time derivative (WEST) provides the alternative structure of atomic states. The most specific feature of WEST theory is the doubling of the space of states. The particle and antiparticle are equivalent in this theory; they are not linked to positive and negative energy states. In contrast to the theories based on the equations with the first time derivative, in this case the particles of the same charge can form the bound states. As a result, the discrete energy spectrum of light atoms consists of the three bands: atomic states (AS) adjacent to upper or down continuum; and nuclear states (NS), i.e. deeply bound states the binding energy for which is close to the particle rest mass. The latter states are the states of the particle inside the nucleus. Such kind of energy spectrum of bound states is due to the doubling of the spatial solutions: the solutions divergent at $r=0$ are taken into account along with the non-divergent ones. The boundary conditions of WEST theory require the finiteness of normalization integral, but the eigenfunction can be divergent at $r=0$ in the same time.

In this report we shall concentrate on the problem of stability regions for heavy and superheavy atoms. The WEST theory shows that in light atoms the binding energy of AS increases and NS decreases with nucleus charge. Hence, the crossing points appear inevitable under nucleus charge variation. These crossing points correspond to critical values of nucleus charge at which the drastic reconstruction both NS and AS occurs. Further increase in nuclear charge may result in decrease of AS binding energy. Along with the crossing the fusion of atomic energy terms occurs. The fusion point determines the boundary value of nucleus charge for stable atom-like ensembles of charged particles.

\section{References}

1. W.B. Kaufmann, P.B. Siegel, W.R. Gibbs, Phys. Rev. C 46, 1474 (1992)

2. P.A.M. Dirac, Proc. Roy. Soc. A 117, 612 (1928)

3. C.G. Darwin, Proc. Roy. Soc. A 118, 654, A120, 621 (1928)

4. W. Gordon, Z. f. Phys. 48, 11 (1928)

5. Ya.B. Zeldovich, V.S. Popov, Physics Uspekhi 105, 403 (1971)

6. A.V. Andreev, Physics Letters A 372 pp 3841-3845 (2008)

7. A.V. Andreev, Relativistic Quantum Mechanics (Moscow: Fizmatlit, 2009) 